\title{
Distributed Algorithms for Convexified Bad Data and Topology Error Detection and Identification Problems
}

\author{
Yang Weng ${ }^{\dagger}$, Marija D. Ilić*, Qiao $\mathrm{Li}^{\ddagger}$, and Rohit Negi* \\ ${ }^{\dagger}$ Department of Civil and Environmental Engineering, Stanford University, Stanford, California 94305, USA, \\ yangweng@stanford.edu, \\ *Department of Electrical and Computer Engineering, Carnegie Mellon University, Pittsburgh, Pennsylvania \\ 15213, USA, \{milic,negi\} @ece.cmu.edu, \\ ${ }^{\ddagger}$ Qualcomm Research New Jersey, New Jersey 08807, USA, liqiao02@gmail.com
}

\begin{abstract}
Large-scale smart grids call for online algorithms that are able to achieve the most accurate estimates. This paper shows how to achieve both the scalability and near globally optimal results for bad data and topology error detection and identification problems, by conducting fully distributed algorithms over convexified problem formulations. The proposed distributed decomposition is realized by 1) reducing a large network into much smaller network "cliques" which do not need extensive information exchange; 2) performing a Lagrangian dual decomposition in each clique and passing messages between cliques; and 3) conducting alternative coordinate descent optimization for robustness. To reduce the relaxation error in the convexification procedure, a nuclear norm penalty is added to approximate original problems. Finally, we propose a new metric to evaluate detection and identification results, which enables a system operator to characterize confidence for further system operations. We show that the proposed algorithms can be realized on IEEE test systems with improved accuracy in a short time.
\end{abstract}

Index Terms-Power systems; bad data and topology error; detection and identification; distributed algorithms; convexification; semidefinite programming

\section{INTRODUCTION}

As one of the most significant infrastructures in human society, the electric power grid not only provides electricity as a form of flexible, convenient energy for industrial and individual uses, but also provides it in a clean and relatively easy way to transmit. Despite 130-plus years of development and engineering, electric power systems are still under intense pressure to achieve stability against outages and blackouts as the power grid moves into large scale network with tons of new devices and loads. While having a potential to reduce the impact on the environment, increase fuel diversity, and bring about economic benefits, new grid components also raise tremendous concerns regarding the secure and reliable operation of the backbone EHV/HV power grids.

Speaking more generally, the power system operators of traditional power grids face significant challenges in managing the effects of small scale generations and loads, which include but not limited to renewable energy generators, such as wind/solar generators; responsive small electricity users; and electricity users which can offer storage to utility, such as electric cars. Their topology and data quality need to be estimated to account for their effects on the state of the backbone power grid. Therefore, highly accurate algorithms capable of dealing with increasing number of bad data and constantly varying network topology are needed.

However, as most bad data and topology identification algorithms currently used by the electric power industry is computationally complex, they are typically used for Extra High Voltage (EHV), High Voltage (HV) and, at times, for Medium Voltage (MV) representation of the complex multivoltage level power grids. This, in turn, makes it very hard to detect and identify bad data and topology error within many new diverse resources and users connected to the Low Voltage (LV) level distribution systems. A multi-layered, distributed implementation of bad data and topology error detection and identification for future electric energy systems is likely to become the preferred approach. This requires a systematic design of distributed algorithms whose performance does not worsen relative to the centralized methods.

In this paper, we first extend the convexification idea in state estimation [1] to bad data and topology error detection ( $L_{2}$ norm) and identification ( $L_{1}$ norm) with substation modeling. The goal is to improve accuracy with a near global optimum estimate by embedding states into proper high dimensional space. This is because there is a strong relationship between SDP formulation for state estimation and the SDP formulation for optimal power flow, e.g., [2], [3] show that the rank-one condition is satisfied with most power grid topologies. However, as a centralized implementation of the semidefinite programming (SDP)-based approach is computationally prohibitive [1], we propose to implement a distributed algorithm based on the underlying power system graph for scalability. The objective decomposition is achieved by using the Lagrangian dual decomposition method [4]. To decompose the system-level positive-semidefinite constraint over the state matrix, the notion of induced chordal graphs is used. Although this is similar to the distributed implementation of the SDP-based AC optimal power flow method [5]-[7], we do not set limits on states, input or output variables due to qualitatively different performance objectives between our problems and AC optimal power flow problem. Then, the proof of equivalence between the centralized algorithm and 
the distributed algorithm is detailed next. Different than past works, this paper proposes how to reduce approximation error in convex-relaxation by penalizing the objective with a nuclear norm instead of a pure rank-one constraint relaxation.

Therefore, by using message exchange on coupling nodes between neighboring local networks, the original centralized SDP detection and identification problems can be characterized in a distributed manner by performing local SDP computation. Obviously, a direct consequence is computational time reduction if parallel processors are used. A fully distributed implementation can also be achieved by using coordinate descent optimization for robustness [8].

In comparison with existing methods, the novelty of the proposed approach lies in 1) past WLS-based bad data and topology error detection and identification have local optimum issues. In this paper, we not only show how to convexify such problems but also show how to conduct them in a distributed way. 2) We show that the distributed computation can be generalized. This means that, in addition to $L_{2}$ norm, $L_{1}$ norm can also be used for error identification. 3) We derive lower bounds to characterize the performance of the arbitrary detection and identification methods. 4) [9] shows results about distributed state estimation based on boundary division, but our results are about distributed bad data and topology detection and identification based on the network structure decomposition, e.g., chordal graphs decomposition. [9] relaxes the rank-one constraint, but we add a nuclear norm penalty to approximate such a constraint.

If implemented, such algorithms are likely to form the basis for "smart" grids by enabling even many small system users to participate in enhancing system operation in predictable ways. For example, the topology (i.e. circuit breaker status) and data quality (i.e. power injection measurements) of smart meters would not have to be estimated by the operator of the backbone system. Instead, topology error and bad data of small users get estimated in a distributed manner by messagepassing with neighboring system users which have smart meters. The aggregated information is then communicated in a bottom-up way to the backbone system operator. Highly accurate distributed detection and identification as well as greatly reduced computational time are illustrated through simulations.

This paper is organized as follows: In Section II, we review detection and identification methods for bad data and topology error; then a convex-relaxation-based approach is proposed to resolve the local optimum issues; in Section III, we propose a distributed SDP-based algorithm; in Section IV, we describe the simulation results for IEEE test systems; finally, we conclude this paper in Section V; an Appendix on using power flow measurements in the distributed algorithm is also included.

\section{REVIEW}

\section{A. Bad Data and Topology Error Detection}

As both bad data and topology error can have a dramatic influence on measurements, they usually cause large residuals in weighted least square minimization (1). Therefore, the resulting objective value is used in a chi-squared test to detect them.

$$
\min _{\boldsymbol{v}} J_{2}(\boldsymbol{v})=\sum_{i=1}^{m}\left|\frac{z_{i}-h_{i}(\boldsymbol{v})}{\sigma_{i}}\right|^{2},
$$

where vector $\boldsymbol{v}=\left(\left|v_{1}\right| e^{j \delta_{1}},\left|v_{2}\right| e^{j \delta_{2}}, \cdots,\left|v_{n}\right| e^{j \delta_{n}}\right)^{T}$ represents the system states to be estimated for error detection. $h_{i}(\cdot)$ relates the unknown state variable $\boldsymbol{v}$ to the $i^{t h}$ noiseless measurement. $z_{i}$ is the $i^{\text {th }}$ telemetered measurement, such as power flow and voltage magnitude. $\sigma_{i}$ is the standard deviation of Gaussian noise $u_{i}$ in $z_{i}$, where noises in different measurements are assumed to be independent. Finally, $m$ represents measurement number.

Next, one looks into a chi-squared distribution table to find a threshold, i.e., $J_{(m-n), 95 \%}$, corresponding to a confidence probability $95 \%$ and $m-n$ degrees of freedom. If $J_{2}(\boldsymbol{v})>J_{(m-n), 95 \%}$, a bad data or topology error is declared [10], [11]. The bus associated with the largest measurement residual is called a suspicious bus. Deciding whether a test failure indicates a sensor error or a topology error is out of the scope for this paper. Interested readers are referred to the Section 8.6.1 of [12] and [10], [11].

\section{B. Bad data and Topology Error Identification}

For bad data identification, one approach is to assume that the measurement associated with the largest residual in (1) to be bad data. So one iterates a chi-squared test with a measurement removal until the test is passed. Another approach is to use Weighted Least Absolute Value (WLAV), or $L_{1}$ norm minimization in (2) to find bad data (sparse noises ) [13] altogether.

$$
\min _{\boldsymbol{v}} J_{1}(\boldsymbol{v})=\sum_{i=1}^{m}\left|\frac{z_{i}-h_{i}(\boldsymbol{v})}{\sigma_{i}}\right| .
$$

For topology identification, one may adjust topology connection associated with large residuals found in (1), but extending suspicious bus into a sub-station model may be a better idea. This approach starts by changing measurement model $z_{i}=h_{i}(\boldsymbol{v})+u_{i}$ into (3) to explicitly account substation circuit breaker status (Chapter 8 of [12]).

$$
z_{i}=h_{i}(\boldsymbol{v})+M_{i} \boldsymbol{f}+u_{i},
$$

where the state $\boldsymbol{v}$ is extended by adding the line power flow vector $f$ through circuit breakers in the suspicious substation. In equation (3), the term $M_{i} f$ represents the effect of these flows in each measurement $z_{i} ; M_{i}$ is an incident matrix defining the interconnection of suspected circuit breakers [12].

To decide the digital status of a circuit breaker, one needs to estimate $f$. One can choose $p=2$ (for detection) or $p=1$ (for identification) in (4) for this purpose, where the parameter $p(p \geq 0)$ is chosen to achieve the desired performance. The goal is to obtain a joint extended state $(\hat{\boldsymbol{v}}, \hat{\boldsymbol{f}})$ that best fits the measurement set $\boldsymbol{z}$ according to the measurement model in (3).

$$
\min _{\boldsymbol{v}, \boldsymbol{f}} J_{p}(\boldsymbol{v}, \boldsymbol{f})=\sum_{i=1}^{m}\left|\frac{z_{i}-h_{i}(\boldsymbol{v})-M_{i} \boldsymbol{f}}{\sigma_{i}}\right|^{p} .
$$


Subsequently, $\hat{f}$ is used to estimate digital status of bus breakers for topology identification.

\section{Local Optimum Problem}

Although we talked about several objectives in (1), (2), and (4), we observe that (4) is a general form that includes the other two as special cases. Therefore, in the rest of the paper, we will focus on (4). The difficulty in solving (4) is the inherent nonlinearity within $h_{i}(\boldsymbol{v})$, which renders the objective in the general form to be non-convex. This causes the local optimum issue, which leads to incorrect bad data and topology error detection and identification processes.

\section{Convexification in Bad Data and Topology Error Detection and Identification}

As many measurements can be represented in pairwise products of voltages, using these products can turn the objective into a convex quadratic form. This is called lifting transformation of states. The idea behind this transformation is to embed states into a high dimensional space to enjoy better linearity. For example, [14] tries to lift voltage state to create an intermediate state vector, leading to a non-iterative state estimation algorithm. However, writing pairwise products of voltages and defining new variables in a vectorization form will lose the correlations between different products. Therefore, we aim at preserving the inter-correlation in a matrix form for lifting via $W \triangleq \boldsymbol{v} \boldsymbol{v}^{H}$ [1], [9], [15], [16], where $H$ is the Hermitian operator.

A complex-valued power injection without considering the substation topology can be expressed as

$$
\begin{aligned}
\boldsymbol{s}_{\text {inj }} & =\operatorname{diag}\{\boldsymbol{v}\} \boldsymbol{i}^{H}=\operatorname{diag}\left\{\boldsymbol{v} \boldsymbol{v}^{H} Y^{H}\right\} \\
& =\operatorname{diag}\left\{W Y^{H}\right\}=\operatorname{Tr}\left(W Y^{H}\right) .
\end{aligned}
$$

Since $p_{\text {inj }}=\frac{1}{2}\left(s_{\text {inj }}+s_{\text {inj }}^{H}\right)$, we have

$$
\begin{aligned}
p_{\text {inj }} & =\frac{1}{2}\left[\operatorname{Tr}\left(W Y^{H}\right)+\operatorname{Tr}\left(Y W^{H}\right)\right] \\
& =\frac{1}{2}\left[\operatorname{Tr}\left(W Y^{H}\right)+\operatorname{Tr}(Y W)\right] \\
& =\frac{1}{2}\left[\operatorname{Tr}\left(Y^{H} W\right)+\operatorname{Tr}(Y W)\right] \\
& =\frac{1}{2}\left[\operatorname{Tr}\left(\left(Y^{H}+Y\right) W\right)\right] \\
& =\frac{1}{2}\left[\operatorname{Tr}\left(T^{\mathrm{act}} W\right)\right],
\end{aligned}
$$

where $T^{\text {act }} \triangleq \frac{1}{2}\left(Y^{H}+Y\right)$. Similarly, we define $T^{\text {rea }}=$ $\frac{1}{2}\left(Y^{H}-Y\right)$ and obtain the reactive power balance equations

$$
\boldsymbol{q}_{\text {inj }}=\operatorname{Tr}\left(\left\{T^{\mathrm{rea}} W\right\}\right) .
$$

Therefore, the $i^{\text {th }}$ real and reactive power injections are $p_{\text {inj }, \mathrm{i}}=\sum_{k=1}^{n} T_{i k}^{\mathrm{act}} W_{k i}$ and $q_{\mathrm{inj}, \mathrm{i}}=\sum_{k=1}^{n} T_{i k}^{\mathrm{rea}} W_{k i}$. If the measurements of real and reactive power injections are used for the error detection and identification, (4) turns into

$$
\begin{aligned}
\underset{W, \boldsymbol{f}}{\operatorname{minimize}} \sum_{i=1}^{n} & {\left[\left(\sum_{k=1}^{n} T_{i k}^{\mathrm{act}} W_{k i}-z_{i}^{\mathrm{act}}-M_{i} \boldsymbol{f}\right)^{2}\right.} \\
& \left.+\left(\sum_{k=1}^{n} T_{i k}^{\mathrm{rea}} W_{k i}-z_{i}^{\mathrm{rea}}-M_{i} \boldsymbol{f}\right)^{2}\right]
\end{aligned}
$$

subject to $W \succeq 0, \operatorname{rank}(W)=1$.

By relaxing the rank-one constraint, a convexified optimization for bad data detection and identification has the following form:

$$
\begin{aligned}
\underset{W, \boldsymbol{f}}{\operatorname{minimize}} \sum_{i=1}^{n} & {\left[\left(\sum_{k=1}^{n} T_{i k}^{\mathrm{act}} W_{k i}-z_{i}^{\mathrm{act}}-M_{i} \boldsymbol{f}\right)^{2}\right.} \\
& \left.+\left(\sum_{k=1}^{n} T_{i k}^{\mathrm{rea}} W_{k i}-z_{i}^{\mathrm{rea}}-M_{i} \boldsymbol{f}\right)^{2}\right]
\end{aligned}
$$

subject to $W \succeq 0$.

Thus, problems (1), (2), and (4) are successfully converted into a convex optimization problem, leading to the global optimal solutions for individual bad data and topology error detection and identification problems. Examples of using other measurements for bad data and topology error detection and identification are described in the Appendix A. For illustrative purposes, we formulate the distributed algorithm next when power injection measurements are given.

\section{Distributed IMPLEMENTATION FOR BAD DATA AND TOPOLOGY ERROR DETECTION AND IDENTIFICATION}

In this section, we describe how to use Lagrangian dual method [4] and chordal graph properties for problem decomposition in (7).

\section{A. Objective Decomposition}

The objective in (7) is nonlinear with respect to $W$. We rewrite (7) into an equivalent form

$$
\begin{array}{ll}
\underset{W, \boldsymbol{f}}{\operatorname{minimize}} & \sum_{i=1}^{n}\left(y_{i}^{\text {act }}\right)^{2}+\sum_{i=1}^{n}\left(y_{i}^{\text {rea }}\right)^{2} \\
\text { subject to } & W \succeq 0,
\end{array}
$$

where

$$
\begin{aligned}
y_{i}^{\mathrm{act}} & =\sum_{k=1}^{n} T_{i k}^{\mathrm{act}} W_{k i}-z_{i}^{\mathrm{act}}-M_{i}^{\mathrm{act}} \boldsymbol{f}, \\
y_{i}^{\mathrm{rea}} & =\sum_{k=1}^{n} T_{i k}^{\mathrm{rea}} W_{k i}-z_{i}^{\mathrm{rea}}-M_{i}^{\mathrm{rea}} \boldsymbol{f} .
\end{aligned}
$$

The Lagrangian of the problem above is

$$
\begin{aligned}
& L\left(W, \boldsymbol{f}, \boldsymbol{\lambda}^{\text {act }}, \boldsymbol{\lambda}^{\text {rea }}, \boldsymbol{y}^{\text {act }}, \boldsymbol{y}^{\text {rea }}\right) \\
& =\sum_{i=1}^{n}\left[\left(y_{i}^{\text {act }}\right)^{2}+\lambda_{i}^{\text {act }}\left(y_{i}^{\text {act }}-\sum_{k=1}^{n} T_{i k}^{\text {act }} W_{k i}+z_{i}^{\text {act }}+M_{i}^{\text {act }} \boldsymbol{f}\right)\right] \\
& +\sum_{i=1}^{n}\left[\left(y_{i}^{\text {rea }}\right)^{2}+\lambda_{i}^{\text {rea }}\left(y_{i}^{\text {rea }}-\sum_{k=1}^{n} T_{i k}^{\text {rea }} W_{k i}+z_{i}^{\text {rea }}+M_{i}^{\text {rea }} \boldsymbol{f}\right)\right] .
\end{aligned}
$$


Therefore, the dual problem of the optimization problem in (8) is: $\max _{\boldsymbol{\lambda}} \min _{W, \boldsymbol{f}, \boldsymbol{y}} L\left(W, \boldsymbol{f}, \boldsymbol{\lambda}^{\text {act }}, \boldsymbol{\lambda}^{\text {rea }}, \boldsymbol{y}^{\text {act }}, \boldsymbol{y}^{\text {rea }}\right)$, subject to $W \succeq 0$. Specifically, the dual problem has the following form

$$
\begin{aligned}
& \max _{\boldsymbol{\lambda}} \min _{W, \boldsymbol{f}, \boldsymbol{y}} \sum_{i=1}^{n}\left[\left(y_{i}^{\mathrm{act}}\right)^{2}+\left(y_{i}^{\mathrm{rea}}\right)^{2}+\lambda_{i}^{\mathrm{act}}\left(y_{i}^{\mathrm{act}}+z_{i}^{\mathrm{act}}+\right.\right. \\
& \left.\left.M_{i}^{\text {act }} \boldsymbol{f}\right)+\lambda_{i}^{\text {rea }}\left(y_{i}^{\text {rea }}+z_{i}^{\text {rea }}+M_{i}^{\text {rea }} \boldsymbol{f}\right)\right]-\sum_{i=1}^{n} \sum_{k=1}^{n} N_{i k} W_{k i}
\end{aligned}
$$

subject to $W \succeq 0$,

where

$$
N_{i k} \triangleq \lambda_{i}^{\mathrm{act}} T_{i k}^{\mathrm{act}}+\lambda_{i}^{\mathrm{rea}} T_{i k}^{\mathrm{rea}} .
$$

To remove the nonlinear terms $\left(\left(y_{i}^{\text {act }}\right)^{2}+\left(y_{i}^{\text {rea }}\right)^{2}\right)$, we observe that the objective has a piecewise quadratic form over $y_{i}^{\text {act }}$ and $y_{i}^{\text {rea }}$. The minimum value of the objective is achieved when $y_{i}^{\text {act }}=-\frac{\lambda_{i}^{\text {act }}}{2}$, and $y_{i}^{\text {rea }}=-\frac{\lambda_{i}^{\text {rea }}}{2}$. Therefore, the dual problem above is simplified into

$$
\begin{aligned}
\max _{\boldsymbol{\lambda}} \min _{W, \boldsymbol{f}} \sum_{i=1}^{n}\left[\lambda_{i}^{\mathrm{act}}\left(z_{i}^{\mathrm{act}}-\frac{\lambda_{i}^{\mathrm{act}}}{4}\right)+M_{i}^{\mathrm{act}} \boldsymbol{f}+\lambda_{i}^{\mathrm{rea} .}\right. \\
\left.\left(z_{i}^{\mathrm{rea}}-\frac{\lambda_{i}^{\mathrm{rea}}}{4}\right)+M_{i}^{\mathrm{rea}} \boldsymbol{f}\right]-\sum_{i=1}^{n} \sum_{k=1}^{n} N_{i k} W_{k i}
\end{aligned}
$$

subject to $W \succeq 0$.

Finally, (7) is converted into (11) with a linear objective over $W$ and $f$. This gain of having a linear objective instead of a quadratic objective comes from performing an extra optimization step over the dual decoupled variables $\lambda_{i} s$.

1) $L_{1}$ norm: The objective decomposition above is for $p=$ 2 in (4). When $p=1$, we use the following reformulation.

$$
\underset{W, \boldsymbol{f}}{\operatorname{minimize}} \sum_{i=1}^{n}\left|y_{i}^{\text {act }}\right|+\sum_{i=1}^{n}\left|y_{i}^{\text {rea }}\right|
$$$$
\text { subject to } W \succeq 0 \text {, }
$$

where,

$$
\begin{aligned}
y_{i}^{\text {act }} & =\sum_{k=1}^{n} T_{i k}^{\text {act }} W_{k i}-z_{i}^{\text {act }}-M_{i}^{\text {act }} \boldsymbol{f}, \\
y_{i}^{\text {rea }} & =\sum_{k=1}^{n} T_{i k}^{\text {rea }} W_{k i}-z_{i}^{\text {rea }}-M_{i}^{\text {rea }} \boldsymbol{f} .
\end{aligned}
$$

The optimization above can be reformulated into

$$
\begin{aligned}
\max _{\boldsymbol{\lambda}} \min _{W, \boldsymbol{f}, \boldsymbol{y}} & \sum_{i=1}^{n}\left[\left|y_{i}^{\mathrm{act}}\right|+\left|y_{i}^{\mathrm{rea}}\right|+\lambda_{i}^{\mathrm{act}}\left(y_{i}^{\mathrm{act}}+z_{i}^{\mathrm{act}}+M_{i}^{\mathrm{act}} \boldsymbol{f}\right)\right. \\
& \left.+\lambda_{i}^{\mathrm{rea}}\left(y_{i}^{\mathrm{rea}}+z_{i}^{\mathrm{rea}}+M_{i}^{\mathrm{rea}} \boldsymbol{f}\right)\right]-\sum_{i=1}^{n} \sum_{k=1}^{n} N_{i k} W_{k i}
\end{aligned}
$$

subject to $\quad W \succeq 0$.

The only difference in this $L_{1}$ norm derivation with respect to $L_{2}$ norm derivation is that we need to calculate $y$ numerically, instead of obtaining an explicit expression as in (11).

\section{B. Constraint Decomposition}

In this section, we show how to decompose the positive semidefinite constraint over $W$ of (7) into equivalent PSD constraints over submatrices [6], [17]-[19]. As some graphtheoretic concepts are necessary, we review some concepts related to chordal graphs.

1) Graph Basics: An undirected graph $G(V, E)$ is with vertex set $V$ and edge set $E \in V \times V$. Two vertices $u, v \in V$ are defined to be adjacent if $(u, v) \in E$ and we assume no self-loops, e.g., $(v, v) \notin E$ for any $v \in V$. A key concept in our decomposition is the graph clique, which is defined based on the "induced subgraph" and the "complete graph". A subgraph $G\left(V^{\prime}, E^{\prime}\right)$ is called an induce subgraph if $V^{\prime} \subseteq V$ and $E^{\prime}=E \cap\left(V^{\prime} \times V^{\prime}\right)$. A complete graph means every pair of vertices forms an edge in $E$. Based on these two special graphs, a clique of a graph is defined as an induced subgraph which is complete. A clique is maximal if its vertices do not constitute a proper subset of another clique. In order to use Theorem III.1, we also need the concept of a chordal graph. A chord in a cycle is an edge connecting two non-consecutive vertices of the cycle. Therefore, a graph is said to be chordal (triangulated) if every cycle of a length exceeding four has a chord.

2) Positive Semidefinite Constraint Decomposition: To motivate the decomposition method of a positive semidefinite constraint, we introduce a graph-based mathematical problem called matrix completion. It is defined as the process of adding entries to a matrix with some unknown or missing values. Without any constraint over the matrix, the unknown elements can be arbitrarily chosen, making matrix completion theoretically impossible. However, if a matrix is positive semidefinite, the matrix can be reconstructed. Mathematically, we are given a fully specified graph $G(V, F)$, which represents the partially known graph $G(V, E)$ with $F \in E$. The goal is to reconstruct $E$. For example, $W$ 's element $w_{i j}$ is defined if and only if edge $\{i, j\} \in F$, so only part of the matrix entries are specified. A completion process aims at finding a fully specified positive semidefinite matrix based on $W$. If we assume that $W_{C_{r} C_{r}}$ is completely specified for all maximal cliques $\left\{C_{r} \subseteq V: r=1,2, \cdots, l\right\}$ of $G(V, F)$, a necessary condition for $W$ to have a PSD matrix completion is that each $W_{C_{r} C_{r}}$ is PSD.

Theorem III.1. (Grone et al. [17]) Any partial symmetric matrix $W$ with specified entries $(i, j) \in F$ representing a chordal graph, has a positive semidefinite matrix completion, if and only if all submatrices $W_{C_{r}, C_{r}}(r=1,2, \cdots, l)$ are all PSD.

3) Chordal Graph Generation: To use Theorem III.1 for PSD decomposition, a chordal graph representation is needed. However, the graphs of typical power grids are non-chordal. [18] illustrates how to apply the chordal graph result in Theorem III.1 for a non-chordal graph. The key idea is about fill-in, or a triangulation of a graph $G(V, F)$ by adding virtual edges. In other words, one needs to fill-in the unknown matrix terms to generate cliques. While the problem of finding a fill- 
in ordering that minimizes the total fill-in number is $\mathcal{N} \mathcal{P}$ complete, heuristic methods are available [18].

For example, Cholesky factorization $W=L L^{H}$ can be used, because $W$ is positive semidefinite and the sparsity pattern of $L$ is determined by the sparsity pattern of the matrix $W$. If we define a graph $G(V, F)$ based on $L$, we will have $F \supseteq E$, Therefore, the added edges in $F \backslash E$ correspond to the fill-ins, making graph $G(V, F)$ a chordal extension of the underlying power grid graph $G(V, E)$. Finally, upon generating the chordal graph for a power grid, one needs to search all maximal cliques within it [20]. So, based on the maximal cliques, we finally reach the point of decomposing the positive semidefinite constraint of $W$ into many smaller semidefinite constraints of its submatrices.

In the following, we use an example to illustrate this process. Define a matrix $A$ as follows

$$
W=\left[\begin{array}{cccc}
4 & 0 & 2 & 2 \\
0 & 3 & 2 & -2 \\
2 & 2 & 6 & 0 \\
2 & -2 & 0 & 6
\end{array}\right]
$$

By Cholesky decomposition,

$$
\begin{aligned}
W=L \times L^{T}= & {\left[\begin{array}{cccc}
2.0000 & 0 & 0 & 0 \\
0 & 1.7321 & 0 & 0 \\
1.0000 & 1.1547 & 1.9149 & 0 \\
1.0000 & -1.1547 & 0.1741 & 1.9069
\end{array}\right] } \\
& \times\left[\begin{array}{cccc}
2.0000 & 0 & 0 & 0 \\
0 & 1.7321 & 0 & 0 \\
1.0000 & 1.1547 & 1.9149 & 0 \\
1.0000 & -1.1547 & 0.1741 & 1.9069
\end{array}\right]^{T}
\end{aligned}
$$

The graph $G(V, F)$ based on the lower triangle of $L$ has one more edge than the graph $G(V, E)$ based on matrix $W$. As $F \supseteq E$, the added edge belongs to $F \backslash E$, which is a virtual line connecting bus 2 and bus 3. Finally, there are two cliques in $L$ by looking into its structure. One clique is associated with bus $\{1,3,4\}$ while the other is associated with bus $\{2,3,4\}$. So, the constraint of $W$ being positive semidefinite is equivalent to the following two positive semidefinite constrains.

$$
W_{1}=\left[\begin{array}{lll}
4 & 2 & 2 \\
2 & 6 & 0 \\
2 & 0 & 6
\end{array}\right] \succeq 0, W_{2}=\left[\begin{array}{ccc}
3 & 2 & -2 \\
2 & 6 & 0 \\
-2 & 0 & 6
\end{array}\right] \succeq 0 .
$$

Remark III.2. So far, the discussion of the constraint decomposition is solely based on the structure of $W$, which is unknown in our problem formulation. Nevertheless, as $W$ lies in the objective with an inner product by the system structure matrix $N$ in (10), one can extract the structure of $W$ via $N$ [18].

\section{Convexification-Based Distributed Algorithm for Bad Data and Topology Error Detection and Identification}

Next, we summarize convexification-based distributed SDP computing steps by the following theorem for (4).

Theorem III.3. Optimization of (7) is the same as the following distributed algorithm:

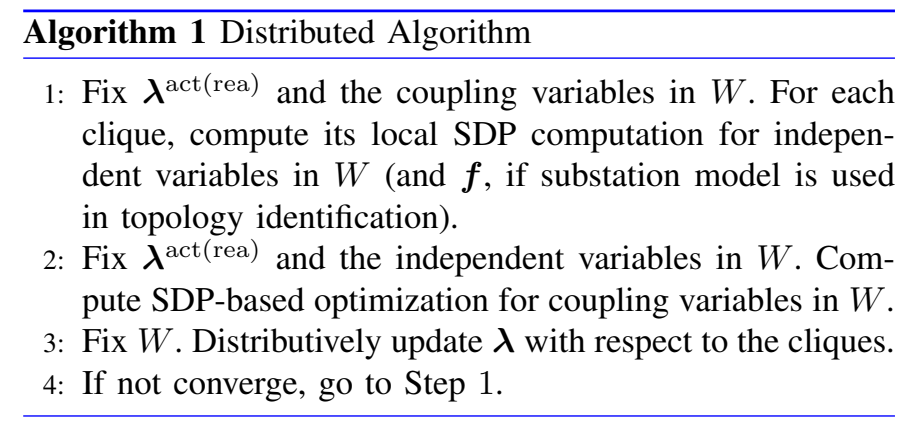

Proof: In the "Lagrangian dual decomposition method", each iteration aims at maximizing the primal variable (matrix $W$ ) while fixing the Lagrangian multiplier $\boldsymbol{\lambda}$. In our proposed parallel approach, this step is replaced by two maximization steps alternating between the independent variables and the coupling variables in $W$. This is because we can regard the optimization over $W$ to be another primal decomposition problem according to [4]. The $i$ th subproblem is:

$$
\min _{W_{i}} f_{i}\left(W_{i}, W_{c}\right)
$$

where $W_{i}$ represents the local variables in the $i^{t h}$ area, and $W_{c}$ is the set of coupling (boundary) variables in $W$. With fixed $W_{c}$, the $i$ th subproblem is assumed to have an optimal value $\phi_{i}\left(W_{c}\right)$. Then the original problem is equivalent to the master problem

$$
\min _{W_{c}} \sum_{i} \phi_{i}\left(W_{c}\right)
$$

with variable set $W_{c}$. This problem is called primal decomposition since the master problem manipulates primal (complicating) variables. As the original problem is convex, so is the master problem. Therefore, the two-step optimization is equivalent to the one-step optimization over $W$, thus leading to a legitimate decomposition.

1) Adding a Convex Penalty Function in Local Optimization: One drawback of the convex relaxation approach is the removing of the rank-one constraint in (7). One can only hope for luck that a low-rank solution will be obtained. As a high-rank solution will lead to deteriorated performance in error detection and identification, we propose to control the resulting solution by penalizing the objective for having a low-rank approximation solution at each subproblem while keeping the convexity. As the rank-one constraint is nonconvex, we introduce the nuclear norm to keep the convexity while approximating the rank-one constraint [21]. This leads to a low-rank solution for $W$. Since we can not minimize the rank function directly due to its non-convexity, we add a nuclear norm to (14) to approximate the rank-one constraint.

$$
\min _{W_{i}} f_{i}\left(W_{i}, W_{c}\right)+\lambda\left\|W_{i}\right\|_{*},
$$

where $\lambda$ is a free weight parameter and

$$
\left\|W_{i}\right\|_{*}=\sum_{j=1}^{l} \gamma_{j}\left(W_{i}\right)
$$

where $\gamma_{j}\left(W_{i}\right)$ is the $j^{t h}$ eigenvalue of the submatrix $W_{i}$. The connection between the rank function and the nuclear norm 
can be seen via the following inequality (see [22], [23]), which holds for any matrix of at most rank $r$,

$$
\left\|W_{i}\right\|_{*} \leq \sqrt{r}\left\|W_{i}\right\|_{F} .
$$

where the subscript $F$ represents Frobenius norm. In fact, the nuclear norm is the convex envelope of the rank function on the set $\left\{W_{i}:\left\|W_{i}\right\|_{F} \leq 1\right\}$.

In the new objective (16), we have a $p$-norm $(\mathrm{p}=1$ or 2$)$ and a nuclear norm functions over $W_{i}$. As norm functions are convex and a non-negative combination of convex functions leads to another convex function [24], the objective in (16) is convex. As a result, the new problem is still a convex optimization problem, which enforces low-rank solutions.

\section{Fully Distributed Algorithm for Robustness}

The parallel algorithm in section III-C needs coupling variables to be computed together, which limits the ability to deploy bad data and topology error detection and identification in a fully distributed way. To enable fully distributed computing of coupling variables, we introduce coordinate descent method, which is based on the idea that the minimization of a convex multivariable function $f(W)$ can be realized by minimizing it along one direction (one area) at a time [8]. As the objective is convex, one can achieve the global optimum by fixing the descent direction at the outset, instead of varying descent direction according to a gradient. The key to this result is the separability of the objective function. For instance, one chooses some basis as the search directions: $W_{1}, W_{2}, \cdots, W_{n}$, where $W_{i}$ includes the independent variables and coupling variables in the $i^{\text {th }}$ clique. Then, we cyclically iterate through each direction to minimize the objective function with respect to that coordinate direction. It follows that, if $\boldsymbol{x}^{k}$ is given, the $i^{\text {th }}$ coordinate of $\boldsymbol{x}^{k+1}$ is given by

$$
W_{i}^{k+1}=\arg \min _{b \in R} f\left(W_{1}^{k+1}, \cdots, W_{i-1}^{k+1}, b, W_{i+1}^{k}, \cdots, W_{n}^{l}\right) .
$$

Therefore, a fully distributed SDP computation can be achieved by locally updating the clique matrix $\left\{\bar{W}_{i}\right\}$, the dual variable $\boldsymbol{\lambda}$ associated with the clique, and optimization of (7) is the same as the following distributed algorithm:

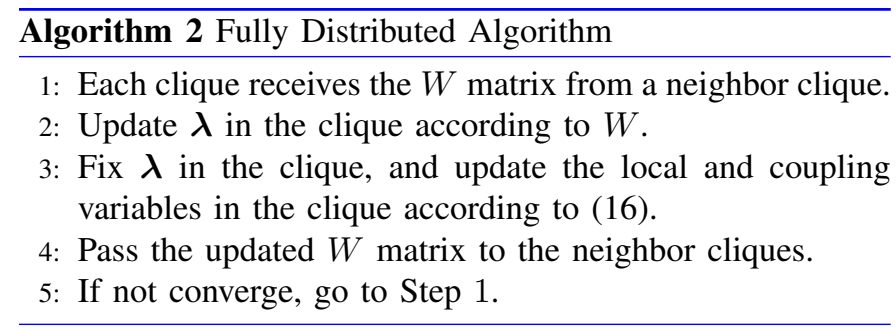

\section{E. The Metric of a Local Confidence.}

Note that, after obtaining the local state matrix $\widehat{W}_{c}$, one can directly compute its local cost function, which has a global minimum smaller than or equal to the global minimum in using $\boldsymbol{x}_{c}$; this provides lower bounds to the local optimization problems in bad data and topology error detection and identification. Therefore, the local system operator can use this lower bound to evaluate the optimality of bad data and topology error detection and identification, leading to a new metric of local estimation confidence for each area.

\section{Simulation Results}

\section{A. Data generation}

The simulations are implemented in the IEEE standard test systems for IEEE 4, 14, 30, 39, 57, 118, and 300 buses. Similar performance improvements are observed. The data has been preprocessed by the MATLAB Power System Simulation Package (MATPOWER) [25], [26]. The measurement set includes the following: (1) the power injection on each bus; (2) the transmission line power flow 'from' or 'to' each bus that it connects; (3) the direct voltage magnitude of each bus and (4) the voltage phase angle of each bus. The measurements are randomly chosen and the total number is around three times the bus number. To obtain measurements, a power flow is first run to generate the true state of the power system, after which Gaussian noises and bad data (or topology error) are added. The Gaussian noises are generated according to standard deviations in Table.I [12].

TABLE I: Standard Deviation for Noise Variables

\begin{tabular}{cc}
\hline \hline Measurement type & Standard deviation for noise variables \\
\hline power injection & $1.5 \%$ \\
power flow & $2 \%$ \\
other types & $1 \%$ \\
\hline
\end{tabular}

Finally, we test our proposed algorithms only when the system is observable.

\section{B. Improved Accuracy}

1) Detection Accuracy: First, the performance of the SDPbased approach is demonstrated in bad data detection scenario, where measurements are corrupted by both Gaussian noise and bad data. One to four bad data are injected into measurements. The error level is set to be above $20 \%$ of the original measurements which were only corrupted by Gaussian noise. Two simulation settings are described in Table II.

\section{TABLE II: IEEE Testing System Details}

\begin{tabular}{ccc}
\hline \hline Bus Number & 14 & 30 \\
\hline No. of power injection measurements & 7 & 12 \\
\hline No. of power flow measurements & 26 & 56 \\
\hline No. of voltage related measurements & 8 & 14 \\
\hline Total measurement number & 41 & 82 \\
\hline Redundancy & 1.58 & 1.41 \\
\hline Number of bad data (larger than $20 \%$ ) & $1,2,3,4$ & $1,2,3,4$ \\
\hline Number of topology error & $1,2,3,4$ & $1,2,3,4$ \\
\hline Gaussian noise level & $5 \%$ & $5 \%$ \\
\hline
\end{tabular}

For example, one simulation for the 14-bus case has the measurement locations, types, and values in Table III and Table IV. The error variance is shown in Table I. Four bad data are added to the power injections at bus 1 and bus 5 power injection, voltage magnitude bus 14 , reactive power flow on the branch $6-13$. 
TABLE III: Measurement values on the buses or branches

\begin{tabular}{ll}
\hline \hline Measurement Type & Bus Numbers or Branch Numbers \\
\hline active power injections & $1,5,14$ \\
reactive power injections & $4,9,10,11$ \\
phase angles & $5,7,13$ \\
voltage magnitudes & $2,5,7,9,14$ \\
active power flows & $1-2,2-4,3-4,4-7,4-9,5-6,6-11$, \\
& $7-8,7-9,9-10,9-14,10-11,12-13,13-14$ \\
reactive power flows & $1-2,1-5,4-5,4-9,5-6,6-11,6-12$, \\
& $6-13,9-10,9-14,10-11,13-14$ \\
\hline
\end{tabular}

TABLE IV: Measurement values on the buses or branches

\begin{tabular}{ll}
\hline \hline Measurement Type & Bus Numbers or Branch Numbers \\
\hline active power injections & $2.2802,-0.0612,-0.1465$ \\
reactive power injections & $0.0392,0.1655,0.0593,0.0163$ \\
phase angles & $-8.7751,-13.6313,-15.6142$ \\
voltage magnitudes & $1.0322,1.0271,1.0611,1.0804,1.0299$ \\
active power flows & $1.5975,0.5512,-0.2297,0.2792,0.1572$, \\
& $0.4416,0.0724,0.0000,0.2829,0.0535$, \\
& $0.0942,-0.0374,0.0162,0.0574$ \\
reactive power flows & $-0.2049,0.0380,0.1523,-0.0944,0.1249$, \\
& $0.0359,0.0257,0.0694,0.0413,0.0359$, \\
& $-0.0163,0.0172$ \\
\hline
\end{tabular}

Then, the distributed SDP-based approach is solved by CVX [27], [28] with the "SEDUMI" package [29]. For example, the extended chordal graph decomposition of 14 bus case is shown in Fig. 1. It shows how the chordal graph decomposition will divide the network into sub-networks for distributed computation. The dotted lines illustrate the conceptual idea of how to generate a chordal graph based on power system network structure. The circles show the chordal decomposition. This decomposition is introduced in (12)-(13) for positive semidefinite constraint decomposition and objective decomposition in (11).

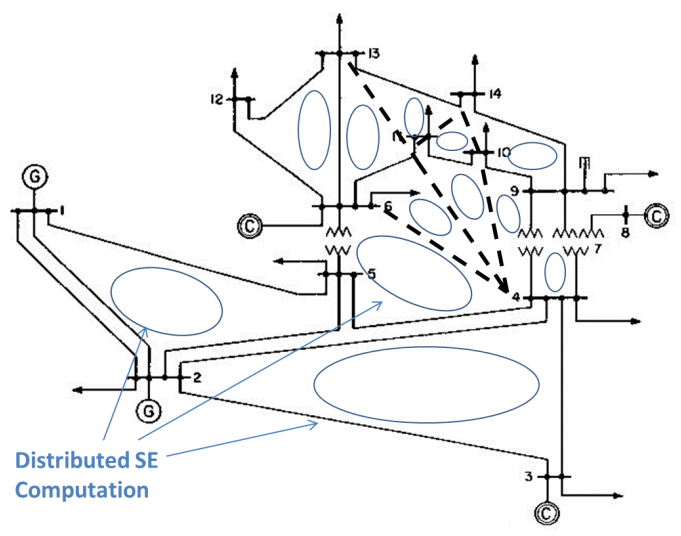

Fig. 1: Extended chordal graph decomposition of IEEE 14 bus system.

After obtaining the state matrix $\widehat{W}$ in (4) with distributed computation in Theorem III.3, the low-rank matrix recovery method is used to obtain an estimate $\hat{\boldsymbol{v}}$ [1]. Then, the chisquared test described in Section II-A is used based on the Weighted Residual Sum of Squares (WRSS) error in (19) for bad data detection or topology error identification.

$$
\text { WRSS } \left.=\sum_{\mathrm{i}=1}^{\mathrm{m}}\left(\left(\mathrm{z}_{\mathrm{i}}-\mathrm{h}(\mathrm{v})\right)\right) / \sigma_{\mathrm{i}}\right)^{2} .
$$

TABLE V: Identification Result

\begin{tabular}{ccccc}
\hline \hline Meas. No. & Type & Bad Data? & Method 1 & Method 2 \\
\hline Meas. 1 & Active power flow & Yes & Yes & Yes \\
Meas. 2 & Active power flow & Yes & Yes & Yes \\
Meas. 16 & Reactive power flow & Yes & Yes & Yes \\
Meas. 34 & Voltage magnitude & No & Yes & No \\
Meas. 36 & Voltage magnitude & Yes & Yes & Yes \\
\hline
\end{tabular}

In Fig. 2, we illustrate one comparison between two sums of squared residuals ( $y$-coordinate) computed from the old approach and the SDP-based approach. A lower bound is also provided to evaluate the performance. As indicated by the $x$ coordinate, such comparison is conducted for five times with different penetration levels of bad data. In particular, black bar represents the WRSS of WLS approach with Newton's method, where a flat start is used for an initialization. The red bar represents the WRSS of the proposed approach, which significantly improves the performance of the old approach. Further, the lower bound in blue shows that the new approach has a result that is near the global optimum, due to the small distance (the new confidence metric) existing between the minimized objective in SDP-based method and the lower bound.

2) Bad Data Identification Accuracy: After bad data detection, bad data identification is conducted according to Section II-B. To make the estimation robust against bad data, the cost function (2) with $p=1$ (WLAV approach) is chosen so that the WLAV problem is solved with a bus-branch model. In Fig. 3, we plot the identification result with the old approach (WLS with Newton's method (flat start) as a solver) and the new convexification-based approach. As there are four errors when implementing the test, the new approach successfully identify all of them. However, the old approach, as shown in Table V incorrectly identifies measurement 34 as a bad data. This may be caused by a local optimum in the WLAV computation with

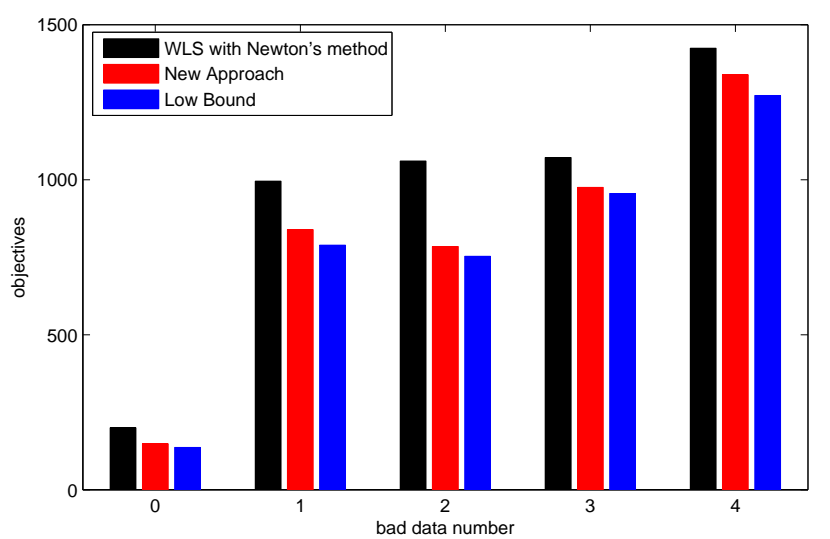

Fig. 2: Sum of squared residuals comparison with a lower bound 


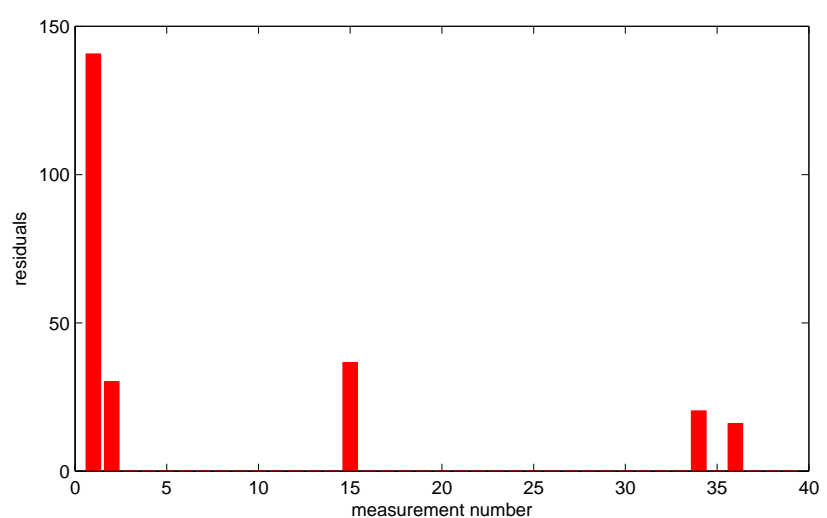

(a) Measurement residuals (old approach)

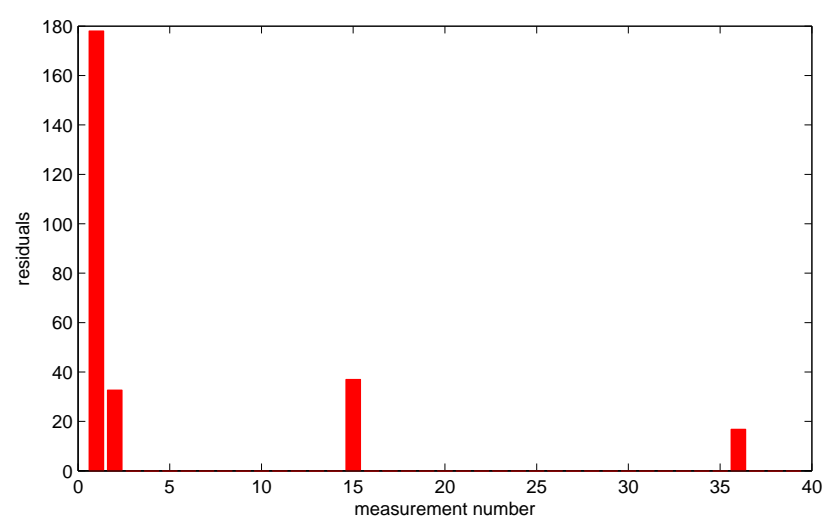

(b) Measurement residuals (new approach)

Fig. 3: One simulation result on IEEE 14 bus with four bad data.

Newton's method. Such a fact leads to a natural interpretation of the identification process: the ability of the new approach to avoid local optimum makes it much closer to the global optimum. This produces a better identification accuracy.

Finally, the averaged performance for bad data identification is displayed in Table VI, where we define the following performance improvement metrics for different rows.

- Bad Data Detection and Topology Error Detection:

$$
\left(\frac{\text { Prob(Successful Detection })_{\text {SDP }}}{\text { Prob(Successful Detection })_{W L S}}-1\right) \times 100 \% \text {. }
$$

- Bad Data Identification and Topology Error Identification:

$$
\left(\frac{\text { Prob }(\text { Successful Identification })_{\mathrm{SDP}}}{\text { Prob(Successful Identification })_{\mathrm{WLS}}}-1\right) \times 100 \% \text {. }
$$

TABLE VI: Averaged Performance Improvements

\begin{tabular}{ccccc}
\hline \hline Goal & 1 Error & 2 Errors & 3 Errors & 4 Errors \\
\hline Bad Data Detection & $11.2 \%$ & $10.2 \%$ & $4.2 \%$ & $5.5 \%$ \\
Bad Data Identification & $2.2 \%$ & $3.7 \%$ & $8.2 \%$ & $22.5 \%$ \\
Topology Error Detection & $5.2 \%$ & $3.9 \%$ & $4.5 \%$ & $4.7 \%$ \\
Topology Error Identification & $11.2 \%$ & $17.0 \%$ & $24.7 \%$ & $32.5 \%$ \\
\hline
\end{tabular}

Remark IV.1. Fig. 2 and Fig. 3 are the results of the proposed decentralized algorithm and they are quite similar to the figures resulting from the centralized algorithm in [16]. This means that our distributed algorithm converges to the centralized algorithm numerically.

3) Topological Error Identification Accuracy: After topology error detection, the bus-branch model is extended to include the detailed substation model [16] ( $f$ is no longer 0 ) of buses with the maximum number of suspicious measurements from which an SDP estimate is obtained. An SDP estimate is obtained by efficiently solving the convex relaxation form of the centralized SDP-based optimization problem. Finally, the estimated power flow states are used to determine the correct topology. While the general accuracy improvement can be seen in Table VI, we use Table VII to show that estimated power flow (SDP method) decreases significantly for the actually open circuit breaker, compared to Newton's method with a flat start. For instance, in an actually open circuit breaker in an IEEE 30-bus test case, the calculated flow estimate (0.0067) of $Q T_{31-36}$ (reactive power flow on the circuit breaker ' $31-36$ '.) for an SDP approach is much smaller than the flow estimate (0.0166) from Newton's method based on a flat start. This prevents miscorrection of topology error. Therefore, the distributed SDP method increases robustness against topology error significantly.

TABLE VII: Estimated Status of Circuit Breakers

\begin{tabular}{cccc}
\hline \hline Circuit Breaker & Power Flow (SDP) & Power Flow (Flat) & Actual State \\
\hline PF 12-32 & 0.0387 & 0.0380 & Closed \\
QT 31-36 & 0.0082 & 0.0166 & Open \\
PF 37-38 & 0.0144 & 0.0317 & Open \\
\hline
\end{tabular}

\section{Computational Time Speedup}

Fig. 4 shows the comparison of computational time in the traditional WLS approach, the centralized SDP approach, and the parallel SDP-based approach over IEEE test systems. Great acceleration can be observed on the proposed distributed algorithm for parallel computing especially for large systems. For these large systems, distributed method saves much more time than the time used to compute the dual variable $\lambda$. Therefore, although the distributed SDP-based approach is more time-consuming than the traditional WLS method, it dramatically reduces the time over the centralized SDP by flatting the curve in Fig. 4. Topology detection has a similar result as the mechanism is the same as discussed in Section II-A.

In Fig. 5, we plot the computational times for bad data identification and topology error identification. In bad data identification, the computational time is based on the optimization with $p=1$. In topology error identification, the computational time is based on the extended bus-branch model. Great computational time acceleration can be seen in both cases from Fig. 5 .

Admittedly, for small systems, such as 4 bus system, the parallel computation requires more time than the distributed computation. This is because, for a small system, dividing the 


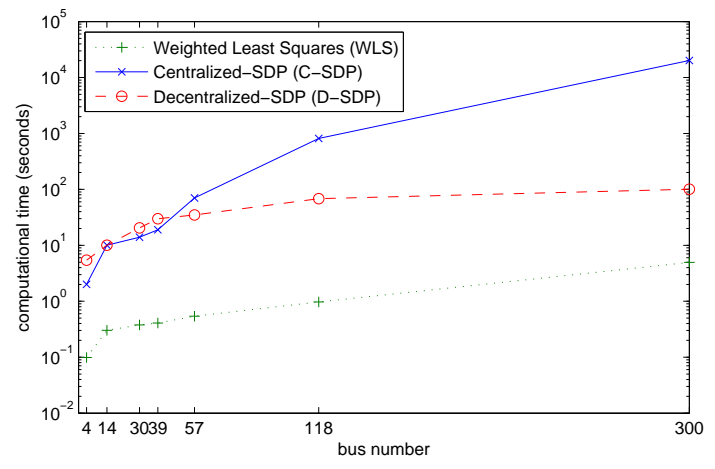

Fig. 4: Computational time comparison for bad data detection.
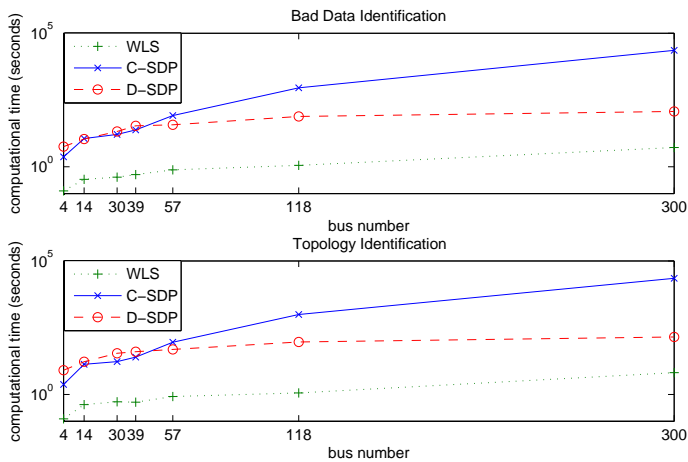

Fig. 5: Computational time comparison for bad data and topology error identification.

network into multiple sub-network cannot substantially lower the optimization variable number. Instead, dual variables' computation may produce heavy burdens to the algorithm. Therefore, Fig. 4 suggests distributed computation for large systems and centralized computation for small systems.

\section{Effect of the Penalty Term}

To understand the penalty term effect over $\operatorname{rank}(W)$, we use Table VIII. The nuclear norm is implemented via the function norm_nuc in the optimization package of CVX.

TABLE VIII: Rank-one approximation by changing the $\lambda$

\begin{tabular}{c|cccc}
\hline \hline$\lambda$ & 0.1 & 1 & 10 & 100 \\
eigenvalue $\gamma_{1}$ & 23.6122 & 8.6978 & 24.1986 & 0.0272 \\
eigenvalue $\gamma_{2}$ & 3.0321 & 0.6183 & 1.2745 & 0 \\
eigenvalue $\gamma_{3}$ & 1.5478 & 0.5670 & 1.1567 & 0 \\
eigenvalue $\gamma_{4}$ & 0.1884 & 0.2544 & 0.0279 & 0 \\
eigenvalue $\gamma_{5}$ & 0.1877 & 0.2482 & 0.0271 & 0 \\
eigenvalue $\gamma_{6}$ & 0.1439 & 0.1023 & 0 & 0 \\
eigenvalue $\gamma_{7}$ & 0.1352 & 0.0955 & 0 & 0 \\
eigenvalue $\gamma_{8}$ & 0.0430 & 0.0170 & 0 & 0 \\
eigenvalue $\gamma_{9}$ & 0.0427 & 0 & 0 & 0 \\
eigenvalue $\gamma_{10}$ & 0 & 0 & 0 & 0 \\
\hline second largest eigenvalue & $12.84 \%$ & $7.11 \%$ & $5.27 \%$ & $0 \%$ \\
\hline largest eigenvalue & & & &
\end{tabular}

From Table VIII, we can see that when penalty is added, not only the number of non-zero eigenvalues decreases but also the ratio between the second largest eigenvalue and the largest eigenvalue decreases. Therefore, the rank-one condition constraint is approximated better via adding the penalty. However, if $\lambda$ is too big, the optimization is changed to a rank minimization problem as the relative weight given to the sum squared residuals of measurements is too small. Crossvalidation can be used to solve this problem for a proper $\lambda$. In our simulation, we choose $\lambda=10$.

\section{CONCLUSIONS}

The need for future smart grid to accurately detect and identify bad data and topology error on-line in the entire electric power grid makes it more difficult to manage all data in a centralized way than in the past. Therefore, we propose to conduct distributed Semidefinite Programming-based bad data detection and topology identification for near globally optimal estimates. By convexifying the original system, a power system graph is decomposed into subgraphs, and minimal information is exchanged between neighbor subgraphs. A nuclear norm penalty are coordinate descent approach are used to reduce rank approximation error and enable fully distributed computation. Finally, significant improvement is shown using several IEEE test systems regarding the computational time reduction and robustness to bad data and topology errors.

\section{ACKNOWLEDGEMENT}

This work was supported in part by US NSF awards 1218823 and 1422193.

\section{APPENDIX}

This section discusses how to include power flow measurements in the proposed distributed algorithm.

\section{A. Power flow measurements}

Similar to the power injection expression (5), the power flow representation without considering substation topology $\left(M_{i} \boldsymbol{f}\right)$ can be formulated as:

$$
s_{i j}=v_{i} i_{i j}^{H}=v_{i}\left(Y_{\mathrm{bra}} \boldsymbol{v}\right)_{k},
$$

where $k$ indicates that the branch, connecting bus $i$ and bus $j$, is the $k^{t h}$ branch in the case file $\left(M_{i} f\right.$ can be easily added when necessary due to its linear operation over the measurement formula.). Therefore, the power flow vectors are:

$$
\begin{aligned}
& \boldsymbol{s}_{\text {flow }}=\operatorname{diag}\left\{D \boldsymbol{v} \boldsymbol{v}^{H} Y_{f}^{H}\right\}=\operatorname{diag}\left\{T \boldsymbol{v} \boldsymbol{v}^{H} Y^{H} T^{H}\right\}, \\
& \boldsymbol{p}_{\text {flow }}=\operatorname{diag}\left\{D \boldsymbol{v} \boldsymbol{v}^{H} M^{\text {act }} T^{H}\right\}, \\
& \boldsymbol{q}_{\text {flow }}=\operatorname{diag}\left\{D \boldsymbol{v} \boldsymbol{v}^{H} M^{\text {rea }} T^{H}\right\},
\end{aligned}
$$

where $D$ is a deterministic matrix mapping the bus voltages onto the branch voltages. Therefore, (7) turns to

$$
\begin{aligned}
\underset{W}{\operatorname{minimize}} & \sum_{i=1}^{l}\left[\left(D_{i,:} T^{\text {act }} W D_{i,:}^{T}-z_{i}^{\text {act.pf }}\right)^{2}\right. \\
& \left.+\left(D_{i,:} T^{\text {rea }} W D_{i,:}^{T}-z_{i}^{\text {rea.qf }}\right)^{2}\right]
\end{aligned}
$$

subject to $W \succeq 0$, 
where $l$ is the branch power flow measurement number. $T_{i,}$ : is the $i^{t h}$ row of matrix $T$.

The SDP form above is equivalent to the following optimization, by adding equality constraints and auxiliary variables $\boldsymbol{y}^{\text {act.pf }}$ and $\boldsymbol{y}^{\text {rea.qf }}$.

$$
\begin{array}{ll}
\min _{W} & \sum_{i=1}^{l}\left[\left(y_{i}^{\text {act.pf }}\right)^{2}+\left(y_{i}^{\text {rea.qf }}\right)^{2}\right] \\
\text { subject to } & W \succeq 0, \\
& y_{i}^{\text {act.pf }}=D_{i,:} W M^{\text {act }} D_{i,:}^{T}-z_{i}^{\text {act.pf }}, \\
& y_{i}^{\text {rea.qf }}=D_{i,:} W M^{\text {rea }} D_{i,:}^{T}-z_{i}^{\text {rea.qf }} .
\end{array}
$$

Its Lagrangian can be written as

$$
\begin{aligned}
& L\left(W, \boldsymbol{\lambda}^{\text {act.pf }}, \boldsymbol{\lambda}^{\text {rea.qf }}, \boldsymbol{y}^{\text {act.pf }}, \boldsymbol{y}^{\text {rea.qf }}\right) \\
= & \sum_{i=1}^{n}\left[\left(y_{i}^{\text {act.pf }}\right)^{2}+\lambda_{i}^{\text {act.pf }}\left(y_{i}^{\text {act.pf }}-D_{i,:} W M^{\text {act }} D_{i,:}^{T}+z_{i}^{\text {act.pf }}\right)\right] \\
+ & \sum_{i=1}^{n}\left[\left(y_{i}^{\text {rea.qf }}\right)^{2}+\lambda_{i}^{\text {rea.qf }}\left(y_{i}^{\text {rea.qf }}-D_{i,:} W W^{\text {rea }} D_{i,:}^{T}+z_{i}^{\text {rea.qf }}\right)\right] .
\end{aligned}
$$

Therefore the dual problem is

$$
\begin{aligned}
\max _{\boldsymbol{\lambda}} \min _{W, \boldsymbol{y}} \sum_{i=1}^{l}[ & \left(y_{i}^{\text {act.pf }}\right)^{2}+\left(y_{i}^{\text {rea.qf }}\right)^{2}+\lambda_{i}^{\text {act }}\left(y_{i}^{\text {act }}+z_{i}^{\text {act }}\right) \\
& +\lambda_{i}^{\text {rea }}\left(y_{i}^{\text {rea }}+z_{i}^{\text {rea }}\right)-D_{i,:} W T^{\text {act }} D_{i,:}^{T} \\
& \left.-D_{i,:} W M^{\text {rea }} D_{i,:}^{T}\right],
\end{aligned}
$$

subject to $W \succeq 0$.

If we define a matrix $M^{\text {flow }}$ such that

$$
\sum_{i=1}^{l} D_{i,:}\left(T^{\mathrm{act}}+T^{\mathrm{rea}}\right) W T_{i,:}^{T}=\sum_{i=1}^{n} \sum_{k=1}^{n} T_{i k}^{\mathrm{flow}} W_{k i} .
$$

the dual problem is equivalent to

$$
\begin{aligned}
\max _{\boldsymbol{\lambda}} \min _{W, \boldsymbol{y}} & \sum_{i=1}^{l}\left[\left(y_{i}^{\text {act.pf }}\right)^{2}+\left(y_{i}^{\text {rea.qf }}\right)^{2}+\lambda_{i}^{\text {act }}\left(y_{i}^{\text {act }}+z_{i}^{\text {act }}\right)\right. \\
& \left.+\lambda_{i}^{\text {rea }}\left(y_{i}^{\text {rea }}+z_{i}^{\text {rea }}\right)\right]-\sum_{i=1}^{n} \sum_{k=1}^{n} T_{i k}^{\text {flow }} W_{k i}
\end{aligned}
$$

subject to $W \succeq 0$.

Such a form is similar to (10), so we can use the same idea after (10) for objective decomposition. Finally, the objective associated with other measurements, such as voltage magnitude and voltage phase angle, can be decomposed similarly.

\section{REFERENCES}

[1] Y. Weng, Q. Li, R. Negi, and M. D. Ilić, "Semidefinite programming for power system state estimation," IEEE Power and Energy Society General Meeting, Jul. 2012.

[2] J. Lavaei and S. H. Low, "Zero duality gap in optimal power flow problem," IEEE Transactions on Power Systems, vol. 27, no. 1, pp. 92-107, Feb. 2012.

[3] M. A. R. Madani and J. Lavaei, "Promises of conic relaxation for contingency-constrained optimal power flow problem," IEEE Transactions on Power Systems, to appear, 2015.
[4] S. Boyd, L. Xiao, A. Mutapcic, and J. Mattingley, "Notes on decomposition methods," 2007. [Online]. Available: http://www.stanford.edu/class/ee364b/notes/decomposition_notes.pdf

[5] X. Bai and H. Wei, "A semidefinite programming method with graph partitioning technique for optimal power flow problems," International Journal of Electrical Power and Energy Systems, vol. 33, pp. 1309-1314, Sep. 2011.

[6] A. Y. S. Lam, B. Zhang, and D. Tse, "Distributed algorithms for optimal power flow problem," Submitted for publication. [Online]. Available: http://arxiv.org/abs/1109.5229

[7] R. A. Jabr, "Exploiting sparsity in sdp relaxations of the opf problem," IEEE Transactions on Power Systems, vol. 27, no. 2, pp. 1138-1139, May 2012.

[8] D. P. Bertsekas, "Nonlinear programming," Athena Scientific, 1995

[9] H. Zhu and G. Giannakis, "Power system nonlinear state estimation using distributed semidefinite programming," IEEE Journal of Selected Topics in Signal Processing, vol. 8, no. 6, pp. 1039-1050, Dec. 2014.

[10] P. W. D. K. A. Clements, "Detection and identification of topology errors in electric power systems," IEEE Transactions on Power Systems, vol. 3, no. 4, pp. 1748-1753, Nov. 1988.

[11] F. F. Wu and W. Liu, "Detection of topology errors by state estimation," IEEE Transactions on Power Systems, vol. 4, no. 1, pp. 176-183, Feb. 1989.

[12] A. Abur and A. G. Exposito, "Power system state estimation: Theory and implementation," CRC Press, Mar. 2004.

[13] W. Kotiuga and M. Vidyasagar, "Bad data rejection properties of weighted least absolute value techniques applied to static state estimation," IEEE Transaction on Power Apparatus and Systems, vol. PAS-101, no. 4, pp. 844-851, Apr. 1982.

[14] B. Fardanesh, "Direct non-iterative power system state solution and estimation," IEEE Power and Energy Society General Meeting, pp. 1-6, Jul. 2012.

[15] H. Zhu and G. B. Giannakis, "Estimating the state of ac power systems using semidefinite programming," Proceedings 43rd North America Power Symposium (NAPS), pp. 1-7, Aug. 2011.

[16] Y. Weng, M. D. Ilic, Q. Li, and R. Negi, "Convexification of bad data and topology error detection and identification problems in ac electric power systems," IET Generation, Transmission \& Distribution, vol. 9, no. 16, pp. 2760-2767, Dec. 2015.

[17] R. Grone, C. R. Johnson, E. M. Sa, and H. Wolkowicz, "Positive definite completions of partial hermitian matrices," Linear Algebra and Applications, vol. 58, pp. 109-124, 1984.

[18] M. Fukuda, M. Kojima, K. Murota, and K. Nakata, "Exploiting sparsity in semidefinite programming via matrix completion i: General framework," Journal of Mathematical Analysis and Applications, vol. 11, no. 3, pp. 647-674, 2000.

[19] K. Nakata, K. Fujisawa, M. Fukuda, M. Kojima, and K. Murota, "Exploiting sparsity in semidefinite programming via matrix completion ii: Implementation and numerical results," Mathematical Programming, vol. 95, no. B, pp. 303-327, 2003.

[20] D. J. Rose, R. E. Tarjan, and G. S. Lueker, "Algorithmic aspects of vertex elimination on graphs," SIAM Journal on Computing, vol. 5, pp. 266-283, 1976.

[21] B. Recht, M. Fazel, and P. A. Parrilo, "Guaranteed minimum-rank solutions of linear matrix equations via nuclear norm minimization," SIAM review, pp. 471-501, 2010.

[22] C. Olsson and M. Oskarsson, "A convex approach to low rank matrix approximation with missing data," Image Analysis. Springer Berlin Heidelberg, pp. 301 - 309, 2009.

[23] G. Golub and C. V. Loan, "Matrix computations," The Johns Hopkins University Press, 1996.

[24] S. Boyd and L. Vandenberghe, Convex Optimization, 2004.

[25] R. D. Zimmerman, C. E. Murillo-Sanchez, , and R. J. Thomas, "Matpower's extensible optimal power flow architecture," IEEE Power and Energy Society General Meeting, pp. 1-7, Jul. 2009.

[26] R. D. Zimmerman and C. E. Murillo-Sanchez, "Matpower, a matlab power system simulation package," http://www.pserc.cornell.edu/ matpower/manual.pdf, Jul. 2010.

[27] M. Grant and S. Boyd, "CVX: Matlab software for disciplined convex programming, version 2.0 beta," http://cvxr.com/cvx, 2013.

[28] — "Graph implementations for nonsmooth convex programs, recent advances in learning and control," Lecture Notes in Control and Information Sciences, Springer, pp. 95-110, 2008.

[29] J. F. Sturm, "Using sedumi 1.02, a matlab toolbox for optimization over symmetric cones," Optimization Methods and Software (Special issue on Interior Point Methods (CD supplement with software)), pp. 625-653, 1999. 